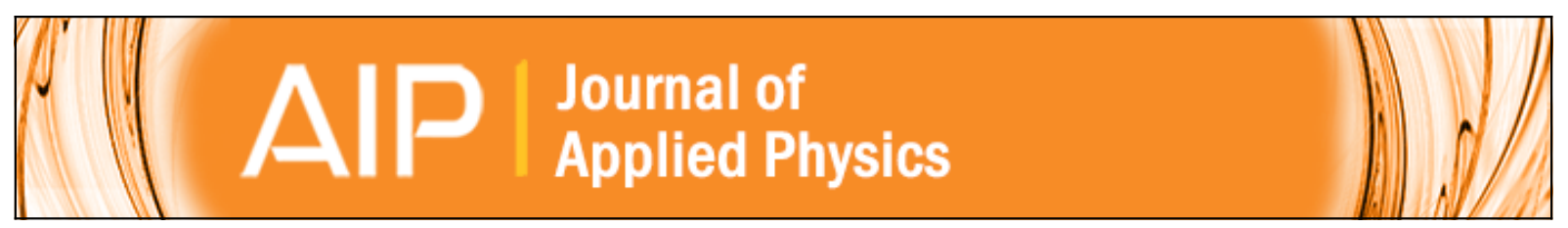

\title{
Effects of laser fluence on silicon modification by four-beam laser interference
}

Le Zhao, Zuobin Wang, Jinjin Zhang, Miao Yu, Siwei Li, Dayou Li, and Yong Yue

Citation: Journal of Applied Physics 118, 233106 (2015); doi: 10.1063/1.4937579

View online: http://dx.doi.org/10.1063/1.4937579

View Table of Contents: http://scitation.aip.org/content/aip/journal/jap/118/23?ver=pdfcov

Published by the AIP Publishing

\section{Articles you may be interested in}

Limitations to laser machining of silicon using femtosecond micro-Bessel beams in the infrared

J. Appl. Phys. 117, 153105 (2015); 10.1063/1.4918669

Periodic antireflection surface structure fabricated on silicon by four-beam laser interference lithography

J. Laser Appl. 26, 012010 (2014); 10.2351/1.4849715

Effects of polarization on four-beam laser interference lithography

Appl. Phys. Lett. 102, 081903 (2013); 10.1063/1.4793752

Subsurface modifications in indium phosphide induced by single and multiple femtosecond laser pulses: A study on the formation of periodic ripples

J. Appl. Phys. 101, 033519 (2007); 10.1063/1.2423136

Photofabrication of wood-pile three-dimensional photonic crystals using four-beam laser interference Appl. Phys. Lett. 83, 608 (2003); 10.1063/1.1595720

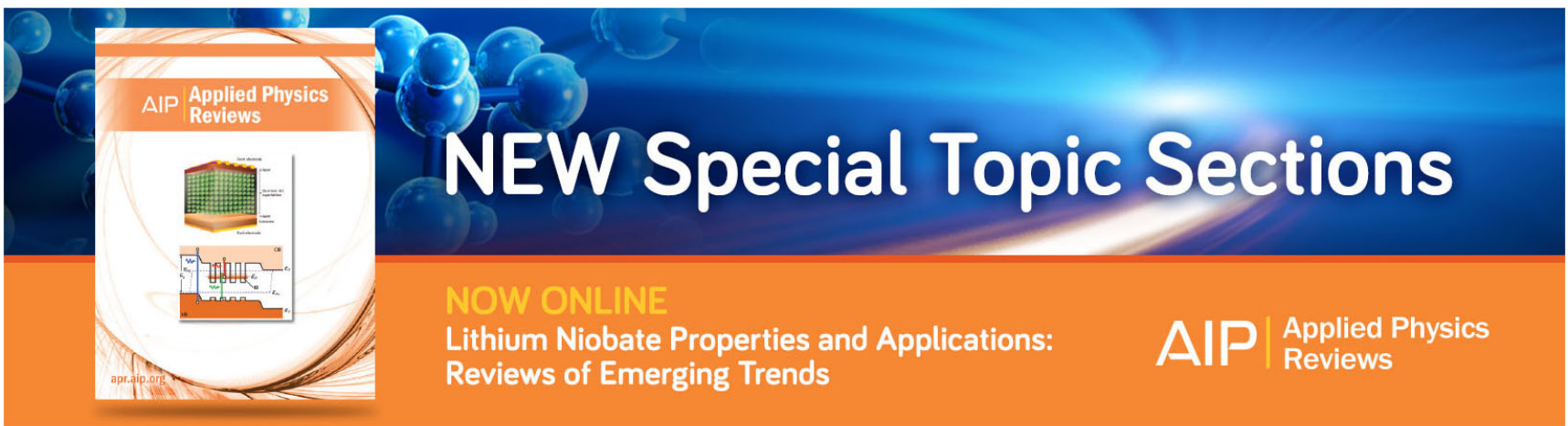




\title{
Effects of laser fluence on silicon modification by four-beam laser interference
}

\author{
Le Zhao, ${ }^{1,2}$ Zuobin Wang, ${ }^{1,2,3, a)}$ Jinjin Zhang, ${ }^{1}$ Miao Yu, ${ }^{1}$ Siwei Li, ${ }^{1}$ Dayou Li, ${ }^{1,2}$ \\ and Yong Yue ${ }^{1,2,3}$ \\ ${ }^{1}$ JR3CN and CNM, Changchun University of Science and Technology, Changchun 130022, China \\ ${ }^{2} J R 3 C N$ and IRAC, University of Bedfordshire, Luton LU1 3JU, United Kingdom \\ ${ }^{3}$ DCSSE, Xi' an Jiaotong-Livepool University, Suzhou 215123, China
}

(Received 3 August 2015; accepted 29 November 2015; published online 17 December 2015)

This paper discusses the effects of laser fluence on silicon modification by four-beam laser interference. In this work, four-beam laser interference was used to pattern single crystal silicon wafers for the fabrication of surface structures, and the number of laser pulses was applied to the process in air. By controlling the parameters of laser irradiation, different shapes of silicon structures were fabricated. The results were obtained with the single laser fluence of $354 \mathrm{~mJ} / \mathrm{cm}^{2}, 495 \mathrm{~mJ} / \mathrm{cm}^{2}$, and $637 \mathrm{~mJ} / \mathrm{cm}^{2}$, the pulse repetition rate of $10 \mathrm{~Hz}$, the laser exposure pulses of 30,100 , and 300 , the laser wavelength of $1064 \mathrm{~nm}$, and the pulse duration of 7-9 ns. The effects of the heat transfer and the radiation of laser interference plasma on silicon wafer surfaces were investigated. The equations of heat flow and radiation effects of laser plasma of interfering patterns in a four-beam laser interference distribution were proposed to describe their impacts on silicon wafer surfaces. The experimental results have shown that the laser fluence has to be properly selected for the fabrication of well-defined surface structures in a four-beam laser interference process. Laser interference patterns can directly fabricate different shape structures for their corresponding applications. (C) 2015 AIP Publishing LLC. [http://dx.doi.org/10.1063/1.4937579]

\section{INTRODUCTION}

Silicon is the basic and important semiconductor material for optical and electronic devices, and the laser processing of silicon wafers is widely used in the fabrication of ICs, processors, memories, sensors and solar cells. Thus, laser interactions with silicon surfaces have been extensively studied and attracted more attention in recent years.

Focused single laser beam processing was widely used for the texturization of silicon surfaces for the fabrication of solar cells, and black silicon with superior absorption property was demonstrated. ${ }^{1}$ Many investigations have been devoted to the processes induced by direct femtosecond (fs), picosecond (ps), and nanosecond (ns) laser processing to produce silicon surface textures for decades. ${ }^{2,3}$ The results are dependent on the laser beam parameters (such as the energy density, pulse duration, wavelength, and pulse repetition rate), and the number of exposure pulses or interaction time and material modification threshold. ${ }^{4,5}$ The defined structures with special features such as ripples and columns can be created with proper selections of the above parameters. ${ }^{5,6}$ The physical phenomena of amorphization, melting, re-crystallization, nucleated vaporization, and ablation were observed during a whole laser processing cycle. ${ }^{5}$

A number of approaches have been made to study the interaction mechanism between focused laser beams and semiconductor materials. Wang et al. investigated the damage thresholds on single-crystals induced by millisecond, nanosecond, and picosecond lasers. ${ }^{7}$ Chichkov et al.

\footnotetext{
a) Author to whom correspondence should be addressed. Electronic mail: wangz@cust.edu.cn
}

presented the theoretical models and explanations of experimental results from femtosecond, picosecond, and nanosecond lasers. ${ }^{8}$ Chichkov et al. reported the ablation of metals by ultrashort laser pulses. ${ }^{9}$ All the above approaches are based on focused laser processing technologies, and time consuming is a significant disadvantage for low-cost mass production due to the laser scanning speed limit.

Compared with focused single laser beam processing, an alternative approach is laser interference lithography technology, which is concerned with the use of interference patterns generated from two or several coherent beams of laser radiation for the modification of materials. ${ }^{10,11}$ Zhang et al. demonstrated periodic antireflection surface structure fabrication on silicon by four-beam laser interference lithography. ${ }^{12}$ Zhao et al. made a silver grating by direct laser interference writing. ${ }^{13}$ Pérez et al. fabricated the submicrometric metallic hollow-core structures by laser interference lithography. ${ }^{14}$ Wang et al. proposed both antireflection and superhydrophobicity structures fabricated by direct laser interference nanomanufacturing. ${ }^{15} \mathrm{Li}$ et al. presented a method for the fabrication of highly ordered superhydrophobic micro-nano dual structures on silicon by direct laser interference lithography. ${ }^{16}$ These approaches have demonstrated that laser interference lithography is a promising technology, which has the advantages of re-configurable patterns and the creation of program controlled structures over large areas for the fabrication of periodic structures on various materials. ${ }^{17}$ However, the effect of laser fluence on silicon modification by laser interference is not adequately addressed considering the heat flow distribution, the radiation effects of laser plasma, and the fluence accumulation 
with the selection of the number of exposure pulses or the interaction time. In addition, the difference of four-beam direct laser interference and single beam laser processing in the heat flow distributions and effects of laser plasma and fluence accumulation have been discussed.

In this work, four-beam laser interference was used to pattern single crystal silicon wafers for the fabrication of surface structures, and the number of laser pulses was applied to the process in air. By controlling the parameters of laser irradiation, different shapes of silicon structures were obtained. The effects of the heat transfer and the radiation of laser interference plasma on silicon wafer surfaces were investigated. The equations of heat flow and radiation effects of laser plasma of interfering patterns in a four-beam laser interference distribution were proposed to describe their impacts on silicon wafer surfaces. The details are described in Sections II-V.

\section{PRINCIPLE}

Interference patterns can be arrays or matrices of laser beam lines or dots with different periods, feature sizes, and pattern shapes. ${ }^{18}$ For two-beam laser interference, the pattern is an array of lines or grating. The intensity distribution of the interference pattern $I$ can be expressed as

$$
I=2 I_{0}[1+\cos (2 k x \sin \theta)],
$$

where $I_{0}$ is the intensity of the two beams, $k=2 \pi / \lambda$ is the wave number, $\lambda$ is the wavelength of the laser, and $\theta$ is the beam incident angle.

The period of the fringe pattern $P$ can be calculated by

$$
P=\frac{\lambda}{2 \sin \theta} .
$$

The pattern period is a function of the wavelength $(\lambda)$ and the incident angle of the beams $(\theta)$. It indicates that the period can be adjusted from nano- to micrometers, which is flexible for many applications.

Fig. 1 shows a configuration of four-beam laser interference and an array of laser dots is formed. The fourbeam interference can be described as the superposition

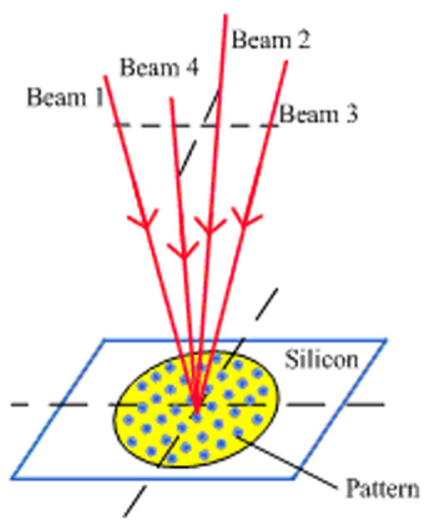

FIG. 1. Schematic diagram of four-beam laser interference. of electric field vectors of four laser beams, and it can be written as

$$
\vec{E}=\sum_{i=1}^{4} \vec{E}_{i}=\sum_{i=1}^{4} A_{i} \vec{P}_{i} \cos \left(k \vec{n}_{i} \cdot \vec{r}_{i} \pm 2 \pi \omega t+\phi_{i}\right)
$$

where $A_{i}$ is the amplitude, $\vec{P}_{i}$ is the polarization vector, $k$ is the wave number, $\vec{n}_{i}$ is the unit vector in the propagation direction, $\vec{r}_{i}$ is the position vector, $\phi_{i}$ is the phase constant, and $\omega$ is the frequency. It can be seen that the interference pattern is a function of the above parameters. In general, the beam intensity determines the peak energy, the incident angle determines the pattern period, and the polarization direction determines the pattern contrast.

The intensity distribution of the interference pattern $I_{m n}$ can be expressed as

$$
I_{m n}=\sum_{i=1}^{4} \sum_{t=1}^{4}\left|\vec{E}_{i}\right|\left|\vec{E}_{t}\right| \cos \left\langle\vec{E}_{i} \cdot \vec{E}_{t}\right\rangle .
$$

Fig. 2 illustrates the principle of four-beam laser interference lithography with three material modification thresholds corresponding to three planes. A particular feature size can be obtained by adjusting the laser pulse energy according to the material modification threshold.

\section{EXPERIMENTAL DETAILS}

The laser interference system used a seeded Q-switched Nd:YAG laser source with the wavelength of $1064 \mathrm{~nm}$, pulse duration of 7-9ns, pulse repetition rate of $10 \mathrm{~Hz}$, and Gaussian beam of $6 \mathrm{~mm}$ in diameter. Two experiments were carried out. One was that the single laser pulses of 354 $\mathrm{mJ} / \mathrm{cm}^{2}, 495 \mathrm{~mJ} / \mathrm{cm}^{2}$, and $637 \mathrm{~mJ} / \mathrm{cm}^{2}$ were used. The other was that the laser pulses of 30,100 , and 300 were used.

In the four-beam interference system, as shown in Fig. 3 , one laser beam was split into four equal parts using beamsplitters and mirrors. Three optical beamsplitters

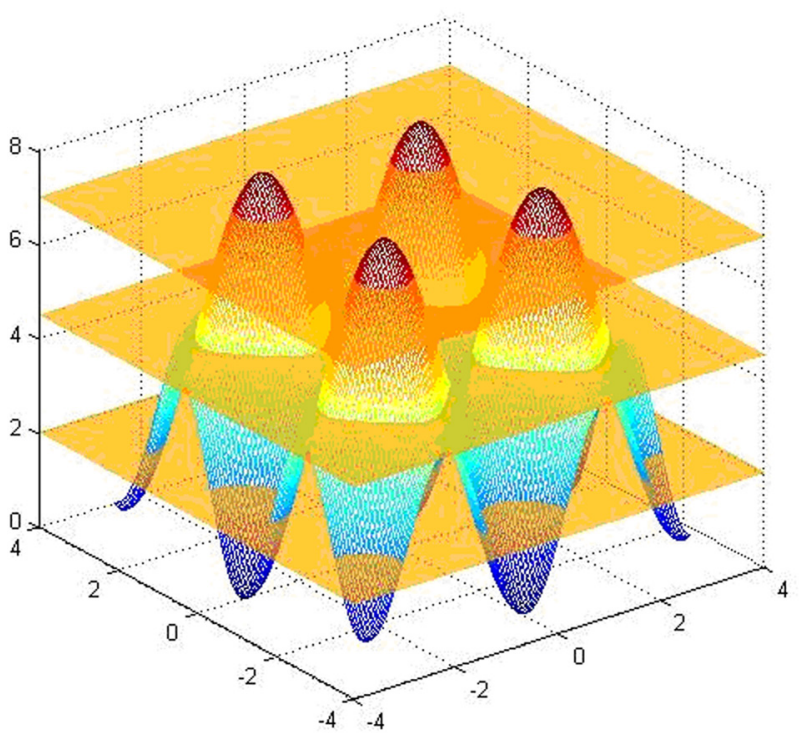

FIG. 2. Principle of four-beam laser interference lithography. 


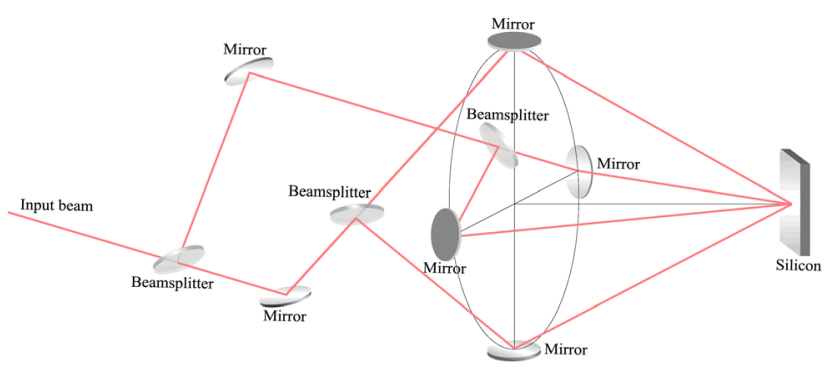

FIG. 3. Schematic diagram of a four-beam laser interference system.

divided the input beam into four noncoplanar coherent beams. A set of mirrors (M1, M2, M3, M4, M5, and M6) steered the beams towards the silicon substrate with the desired angles of incidence $\left(\theta_{1}=\theta_{2}=\theta_{3}=\theta_{4}\right)$. The optical paths of the four beams were identical in order to ensure the spatial overlap of the same parts of the laser spot in the interference field.

The emitted laser fluence of a single pulse was divided into four beams by beamsplitters. The four beams were recombined on the surface of the silicon wafer with specified incident angles, creating periodic interference patterns. The four beams were symmetrically configured in the space distribution with the azimuthal angles of $0^{\circ}, 90^{\circ}, 180^{\circ}$, and $270^{\circ}$. In the experiments, the incident angles of $5.5^{\circ}$ and the polarization angles of $0^{\circ}, 90^{\circ}, 0^{\circ}$, and $90^{\circ}$ were selected. The combination of $1 / 4$ wave plates and polarizers was used to control the pulse energy level of single beams and the polarization direction of each beam. The polarization has a great influence on the intensity distribution of laser interference. ${ }^{19}$ The substrates used in the experiments were single side polished monocrystalline silicon wafers with (100) orientation. After the structures fabricated on the samples, a scanning electron microscope (SEM, FEI XL30) was used to perform the measurements.

\section{RESULTS AND DISCUSSION}

There are two physical models that explain the interactions between the laser and silicon wafer surface in the fabrication process of different structures: the heat transfer model and the radiation effects of laser plasma. ${ }^{20}$ Following the laser radiation, the silicon wafer will be heated, melted, and then vaporized, and several physical processes will also occur, such as oxidation, amorphization, and recrystallization.

For the heat transfer interaction process between the laser and silicon wafer surface, there are two crucial absorption mechanisms, which are intrinsic absorption and freecarrier absorption during the interaction process between the laser and silicon wafer surface depending on the laser and material parameters. ${ }^{7}$ A heat flow expression can describe the laser influence process 8,9

$$
\begin{gathered}
C_{e} \frac{\partial T_{e}}{\partial t}=\frac{\partial}{\partial z} K \frac{\partial}{\partial z} T_{e}-\gamma\left(T_{e}-T_{i}\right)+S \\
C_{i} \frac{\partial T_{i}}{\partial t}=\gamma\left(T_{e}-T_{i}\right),
\end{gathered}
$$

$$
S=I(t) A \alpha \exp (-\alpha z),
$$

where $S$ is the laser heating source term, $T_{e}$ and $T_{i}$ are the temperatures of the electron gas and the lattice, $C_{e}$ and $C_{i}$ are the heat capacities, $K$ is the thermal conductivity, $\gamma$ is the coupling constant, $\tau_{e}=C_{e} / \gamma$ is the electron cooling time, and $\tau_{i}=C_{i} / \gamma$ is the lattice heating time. The spatial model of the incident laser beam is assumed as Gaussian distribution and assumed to increase exponentially with time as $I(t)=I \exp \left(t / \tau_{L}\right)$, where $\tau_{L}$ is the laser pulse duration, $A$ is the surface absorptivity, $\alpha$ is the material absorption coefficient, and $I$ is the intensity distribution. The heat flow expression for the single beam laser influence expressed in Eqs. (5)-(7) can explain the phenomena of laser interaction with silicon wafer such as heat distribution, transfer, and ablation. ${ }^{5-8}$

The intrinsic absorption is the generation of electron-hole pairs when electrons are thermally excited from valence bands into conduction bands. The condition of the intrinsic absorption is that the corresponding photon energy $(h v)$ is greater than the band gap $\left(E_{g}\right)$. In the experiments, the laser wavelength was $1064 \mathrm{~nm}$, the band gap $\left(E_{g}\right)$ of silicon was $1.12 \mathrm{eV}$ at room temperature, and the corresponding photon energy was $h v=h(c / \lambda)=1.17 \mathrm{eV}>1.12 \mathrm{eV}$. The temperatures $T_{e}$ and $T_{i}$ are produced by intrinsic absorption and freecarrier absorption, as shown in Eqs. (5) and (6). The process of laser energy transition inside a silicon wafer can be considered as the generation of carriers, the energy absorption of carriers, and the energy transfer from carriers to lattices. ${ }^{7}$

Since the heating source is the nanosecond laser, and $\tau_{L} \gg \tau_{i}$. It means that the temperatures of the electron gas $T_{e}$ and the lattice $T_{i}$ are equal, i.e., $T_{e}=T_{i}=T$. Hence, Eqs. (5) and (6) can be simplified as ${ }^{8}$

$$
C_{i} \frac{\partial T}{\partial t}=\frac{\partial}{\partial z} K \frac{\partial}{\partial z} T+S,
$$

where $z$ is in the direction perpendicular to the silicon wafer surface. The process of laser interaction with the silicon wafer surface can be described as that the laser pulses first heat the silicon wafer surface to the melt and then to the boiling point. $^{8}$

In the case of four-beam laser interference, the interference pattern consists of a periodic distribution of the intensity. Fig. 2 shows the principle of four-beam laser interference lithography with the intensity distribution from computer simulation. The intensity distribution of interference pattern $I_{m n}$ can be expressed as

$$
I_{m n}=\left[\begin{array}{cccc}
I_{11} & I_{12} & \ldots \ldots & I_{1 n} \\
I_{21} & I_{22} & \ldots \ldots & I_{2 n} \\
\ldots \ldots & \ldots \ldots & \ldots \ldots & \ldots \ldots \\
I_{m 1} & I_{m 2} & \ldots \ldots & I_{m n}
\end{array}\right],
$$

and each element in the matrix can be considered as a laser intensity distribution. The laser interference intensity is assumed to increase exponentially with the exposure time. Hence, the laser interference intensity $I(t)_{m n}$ with the exposure time $t$ can be expressed as ${ }^{9}$ 


$$
\begin{aligned}
I(t)_{m n} & =I_{m n} \exp \left(t / \tau_{L}\right) \\
& =\left[\begin{array}{cccc}
I_{11} & I_{12} & \ldots \ldots & I_{1 n} \\
I_{21} & I_{22} & \ldots \ldots & I_{2 n} \\
\ldots \ldots & \ldots \ldots & \ldots \ldots & \ldots \ldots \\
I_{m 1} & I_{m 2} & \ldots \ldots & I_{m n}
\end{array}\right] \cdot \exp \left(t / \tau_{L}\right),
\end{aligned}
$$

and the laser interference heating source term $S_{m n}$ can be expressed as

$$
\begin{aligned}
S_{m n} & =I(t)_{m n} A \alpha \exp (-\alpha z)=I_{m n} A \alpha \exp \left(-\alpha z+t / \tau_{L}\right) \\
& =\left[\begin{array}{cccc}
I_{11} & I_{12} & \ldots \ldots . & I_{1 n} \\
I_{21} & I_{22} & \ldots \ldots . & I_{2 n} \\
\ldots \ldots & \ldots \ldots & \ldots \ldots & \ldots \ldots . \\
I_{m 1} & I_{m 2} & \ldots \ldots & I_{m n}
\end{array}\right] \cdot A \alpha \exp \left(-\alpha z+t / \tau_{L}\right) .
\end{aligned}
$$

Fig. 4 shows a sectional plot of the four-beam laser interference intensity distribution based on the Gaussian laser interference source. The heat flow expression of the fourbeam laser interference distribution can be written as

$$
C_{i} \frac{\partial T_{m n}}{\partial t}=\frac{\partial}{\partial z_{m n}} K \frac{\partial}{\partial z_{m n}} T_{m n}+S_{m n}
$$

where $T_{m n}$ is the temperature distribution of laser interference and $Z_{m n}$ is the direction perpendicular to the silicon wafer surface. Each interfering dot intensity distribution in an interference pattern can be considered as a heat flow process, and Eq. (12) describes the process of an array of the dot intensity distributions. Different distributions of temperatures and depths with time on a silicon surface can be obtained according to the total heat flow process of four-beam laser interference.

An expression for the ablation threshold fluence after irradiation with $\mathrm{N}$ pulses is related to the single shot ablation threshold $^{5}$

$$
\phi(N)=\phi(1) \cdot N^{\xi-1},
$$

where $\phi(N)$ denotes the modification threshold fluence for $N$ laser pulses, and $\xi$ is a material-dependent coefficient of $0.84 .^{5}$ The diameter of the ablation crater $D$ is related to the maximum laser fluence on the silicon wafer as the following expression: ${ }^{22}$

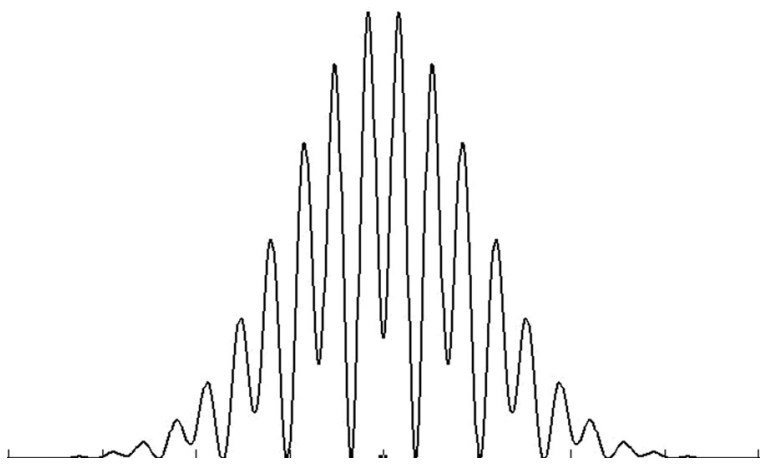

FIG. 4. A sectional plot of the four-beam laser interference intensity distribution.

$$
D^{2}=2 w_{0}^{2} \ln \left(\frac{\varphi_{0}}{\varphi_{t h}}\right)
$$

where $\varphi_{0}$ is the maximum fluence of the Gaussian beam profile, $\varphi_{t h}$ is the ablation threshold fluence, and $w_{0}$ is the beam radius.

Thus, for the four-beam laser interference, Eqs. (13) and (14) can be expressed in the form of matrix as

$$
\begin{gathered}
\varphi_{m n}(N)=\varphi_{m n}(1) \cdot N^{\xi-1}, \\
D_{m n}{ }^{2}=2 w_{0 m n}^{2} \ln \left(\frac{\varphi_{0 m n}}{\varphi_{t h m n}}\right) .
\end{gathered}
$$

An accumulation of energy (i.e., non-complete dissipation of the deposited energy) into silicon wafer has also successfully been employed as $N \cdot \varphi_{m n}(N)$.

For the laser plasma process that affects on the silicon wafer surface, the accumulation of laser energy can cause the increase in the temperature of silicon wafer, break its threshold, and make a small part of the sample melted and gasified. Then, the gaseous silicon can tend to ionize and form the laser plasma. In addition, the laser plasma can absorb more laser energy, becomes high-temperature and high-pressure, and then continuously explodes sharply and compresses the ambient air. ${ }^{21}$

Hence, the generation and expansion of the laser plasma can cause the appearance of laser shock wave, which forms the flower-like silicon structures and the subsequent heat transfer inside the silicon wafer. Each of flower-like silicon structures consists of a silicon hole and many irradiated particles which are homogeneous around the hole, as shown in Fig. 5. The shock wave radius can be written as ${ }^{20}$

$$
R(t)=A\left(\frac{Q}{\rho_{1}}\right)^{\frac{1}{5}} t^{\frac{2}{5}}
$$

where $Q$ is the laser energy, $R$ is the radius of laser shock wave, $t$ is the interaction time, $\rho_{1}$ is the density of surrounding air, and $A$ is the constant related to the air parameters. Thus, in the case of four-beam laser interference, the laser energy $Q_{m n}$ distribution can be expressed as

$$
\begin{aligned}
& Q_{m n}=\left[\begin{array}{cccc}
Q_{11} & Q_{12} & \ldots \ldots & Q_{1 n} \\
Q_{21} & Q_{22} & \ldots \ldots & Q_{2 n} \\
\ldots \ldots & \ldots \ldots & \ldots \ldots & \ldots \ldots \\
Q_{m 1} & Q_{m 2} & Q_{m 3} & Q_{m n}
\end{array}\right]=I_{m n} \cdot S_{\text {Amn }} \cdot T_{\text {Laser }}, \\
& \left.S_{\mathrm{A} m n}=\mid \begin{array}{cccc}
S_{A 11} & S_{A 12} & \ldots \ldots & S_{A 1 n} \\
S_{A 21} & S_{A 22} & \ldots \ldots & S_{A 2 n} \\
\ldots \ldots & \ldots \ldots & \ldots \ldots & \ldots \ldots \\
S_{A m 1} & S_{A m 2} & \ldots \ldots & S_{A m n}
\end{array}\right\rfloor
\end{aligned}
$$

where $T_{\text {Laser }}=N_{\text {laser }} \cdot \tau, N_{\text {laser }}$ is the number of laser pulses, $\tau$ is the pulse duration, $I_{m n}$ is the laser fluence, and $S_{A m n}$ is the area of the laser spot. The four-beam interference shock wave radius can be written as 


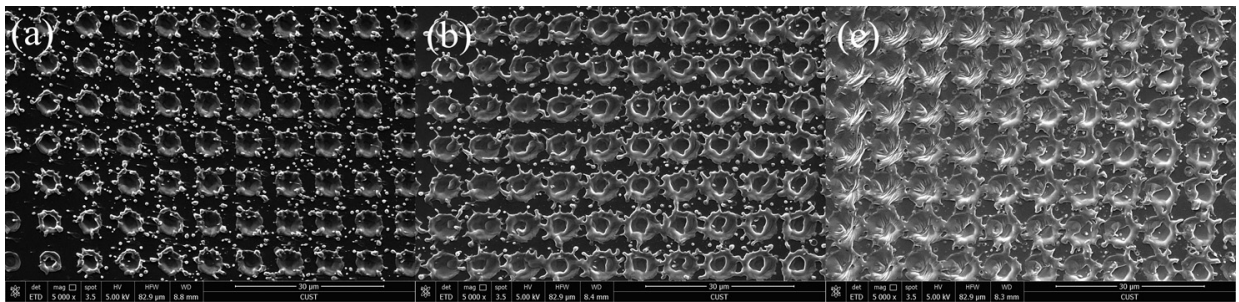

FIG. 5. SEM images of the flower-like silicon structures fabricated by fourbeam laser interference lithography. (a) The single laser fluence of $354 \mathrm{~mJ} /$ $\mathrm{cm}^{2}$, (b) the single laser fluence of $495 \mathrm{~mJ} / \mathrm{cm}^{2}$, and (c) the single laser fluence of $637 \mathrm{~mJ} / \mathrm{cm}^{2}$.

$$
R_{m n}(t)=A\left(\frac{Q_{m n}}{\rho_{1}}\right)^{\frac{1}{5}} t^{\frac{2}{5}}=A\left(\frac{I_{m n} \cdot \mathrm{S}_{\mathrm{Amn}} \cdot T_{\text {Laser }}}{\rho_{1}}\right)^{\frac{1}{5}} t^{\frac{2}{5}} .
$$

Compared to single laser beam processing, an array of multiple laser shock waves can be obtained, and the laser shock wave radius distribution on silicon wafer surfaces can be estimated by Eq. (20).

Fig. 5 shows the SEM images of an array of flower-like silicon structures fabricated by four-beam laser interference lithography with the single laser fluence of $354 \mathrm{~mJ} / \mathrm{cm}^{2}$ (Fig. $5\left(\right.$ a) ), $495 \mathrm{~mJ} / \mathrm{cm}^{2}$ (Fig. 5(b)) and $637 \mathrm{~mJ} / \mathrm{cm}^{2}$ (Fig. 5(c)), and the pulse repetition rate of $10 \mathrm{~Hz}$, the laser wavelength of $1064 \mathrm{~nm}$, and the pulse duration of 7-9 ns. Figs. 5(a)-5(c) show the laser plasma processes with the splashed flowerlike silicon structures around each of silicon holes.

In the case that the laser fluence was $354 \mathrm{~mJ} / \mathrm{cm}^{2}$, an array of flower-like silicon structures on the beam irradiation region was observed on the silicon wafer surface, as shown in Fig. 5(a). Fig. 6 shows the diameter sizes of holes with error bars obtained from different values of laser fluence. The error bars were the average of 10 samples. The average diameter size of the obtained flowers was approximately $6.5 \mu \mathrm{m}$, and each of flowers consists of the silicon hole and many irradiated particles which are homogeneous around the silicon hole. The average diameter size of the holes was about $2.7 \mu \mathrm{m}$ and that of the particles around the silicon holes was about from $300 \mathrm{~nm}$ to $800 \mathrm{~nm}$. When the laser fluence was $495 \mathrm{~mJ} / \mathrm{cm}^{2}$, an array of large flower-like silicon structures was observed, as shown in Fig. 5(b). The average diameter size of flowers was increased to about $7.5 \mu \mathrm{m}$, that of the holes was increased to about $3.8 \mu \mathrm{m}$, and the particles were distributed around the hole on the silicon wafer surface.

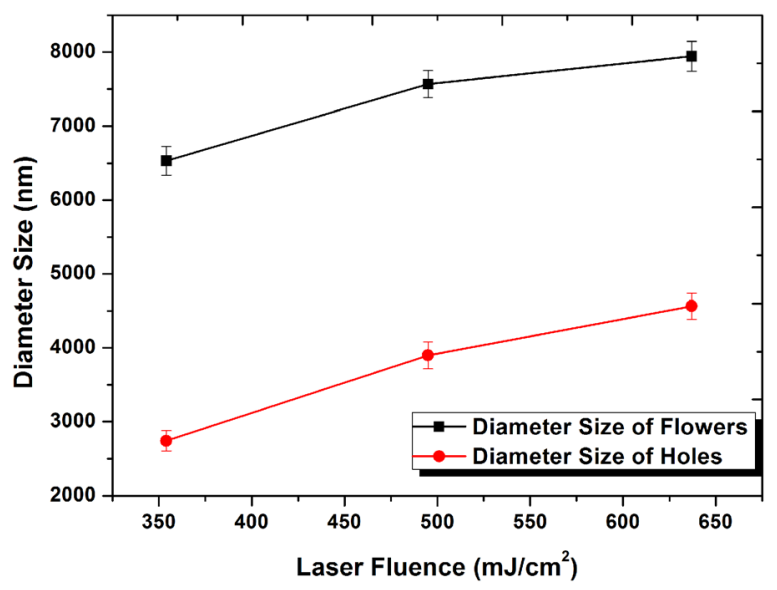

FIG. 6. Diameter sizes of holes with error bars to the average size obtained from different values of laser fluence.
When the laser fluence was $637 \mathrm{~mJ} / \mathrm{cm}^{2}$, the diameter size of the flowers became larger, as shown in Fig. 5(c). The average diameter size of flowers was about $7.9 \mu \mathrm{m}$, and almost all the particles were connected to the center of the flower. The holes became larger on the silicon wafer surface, and the average diameter size was about $4.5 \mu \mathrm{m}$. Fig. 5 indicates that different values of laser fluence can produce different shapes of flower-like silicon structures. The formation process can be provided by the condition of melting, gasification, and ionization of silicon. ${ }^{20}$ Thus, the particles have splashed around each of silicon holes in the experiment.

Figs. 7-9 show the results obtained from the repetition rate of $10 \mathrm{~Hz}$ and the laser pulses of 30,100, and 300. Each laser fluence for all the samples was $637 \mathrm{~mJ} / \mathrm{cm}^{2}$. The different numbers of laser pulses affect the modifications of silicon surfaces and the evolution processes of structures in laser interference lithography. The images present the laser ablation processes, and the physical processes can be described by Eqs. (9)-(16).

In the case that the laser exposures were 30, an array of holes in the beam irradiation center were observed on the silicon wafer surface, as shown in Figs. 7(a) and 7(b). The average depth and diameter size of holes were about $5.5 \mu \mathrm{m}$ and $5.8 \mu \mathrm{m}$. When the exposures were 100, an array of deep holes was observed on the silicon wafer surface, as shown in Figs. 8(a) and 8(b). The average depth of holes was about $8.9 \mu \mathrm{m}$, and the average diameter size of the holes was about $6.8 \mu \mathrm{m}$. When the exposures were 300 , the depth of the holes became deeper and the diameter size also became larger. An array of cones was formed by the same conditions of fourbeam laser interference, as shown in Figs. 9(a) and 9(b). The average depth of holes was about $13.8 \mu \mathrm{m}$, and the average diameter size was changed to $7.8 \mu \mathrm{m}$. An array of holes was not evident and replaced by an array of cones on the silicon wafer surface due to that the laser interference fluence had reached to the threshold value of silicon wafer. Thus, the surrounding materials of silicon microholes were etched and formed the array of cones on the silicon wafer surface. The

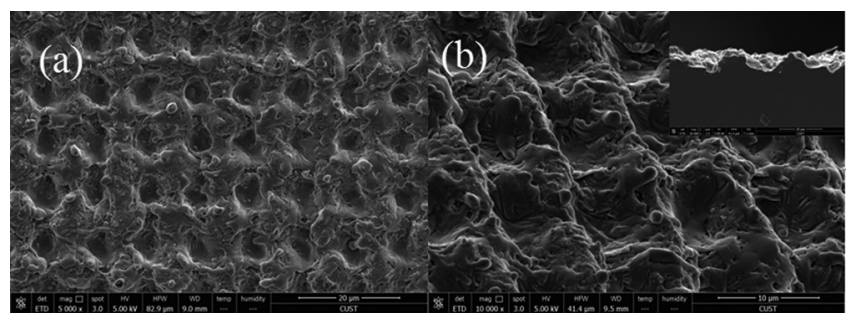

FIG. 7. (a) SEM image $\left(0^{\circ}\right)$ and (b) SEM image $\left(45^{\circ}\right)$ of the pattern centers of the structures fabricated on the silicon wafer surface by four-beam laser interference lithography with 30 laser pulses. 


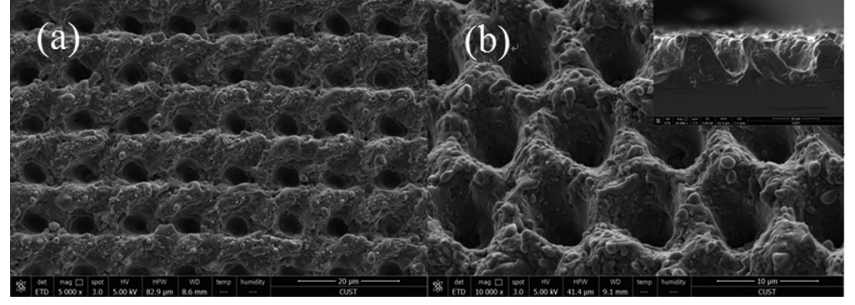

FIG. 8. (a) SEM image $\left(0^{\circ}\right)$ and (b) SEM image $\left(45^{\circ}\right)$ of the pattern centers of the structures fabricated on the silicon wafer surface by four-beam laser interference lithography with 100 laser pulses.

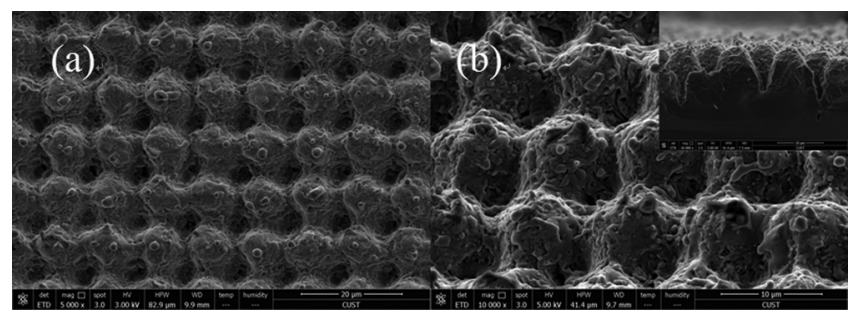

FIG. 9. (a) SEM image $\left(0^{\circ}\right)$ and (b) SEM image $\left(45^{\circ}\right)$ of the pattern centers of the structures fabricated on the silicon wafer surface by four-beam laser interference lithography with 300 laser pulses.

theoretical diameter size on silicon wafer surfaces can be estimated by Eqs. (15) and (16) in the laser ablation process. Figs. 7-9 were the SEM images obtained in the beam irradiation center, and the theoretical diameter size was accordingly calculated. The theoretical diameters were obtained as $3.8 \mu \mathrm{m}, 5.3 \mu \mathrm{m}$, and $9.1 \mu \mathrm{m}$.

Figs. 7-9 show the evolution process of the structures fabricated by four-beam laser interference on the silicon wafer surface. The process was due to the increase in the exposure pulses. It means that the laser energy accumulation is the result of the increase in laser impact on the silicon wafer surface. The increases in the depth and the diameter of the holes are due to that the four-beam laser interference intensity is higher than the silicon material threshold, as shown in Figs. 2, 10, and 11. Figs. 10 and 11 show that different energy accumulation of pulses can meet different demands of material modification thresholds for different shapes of features. Thus, different laser pulses can also affect the morphology of laser interference structures on the silicon wafer surface. Fig. 12 shows theoretical and experimental results for the comparison of their radius sizes with laser pulses.

(a)
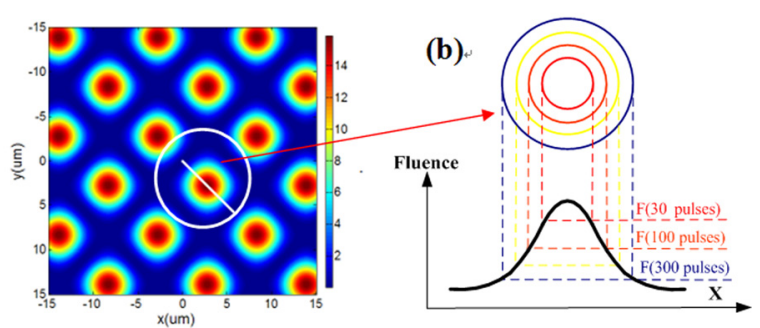

FIG. 10. (a) The computer simulation of intensity distribution of four-beam laser interference, the incident angle was $5.5^{\circ}$, the azimuthal angles were $0^{\circ}$, $90^{\circ}, 180^{\circ}$, and $270^{\circ}$, and the polarization angles were $0^{\circ}, 90^{\circ}, 0^{\circ}$, and $90^{\circ}$ and (b) the schematic profile of a single positive peak dot intensity distribution after the effect of four-beam interference with the laser pulses of 30 , 100 , and 300 . (a)

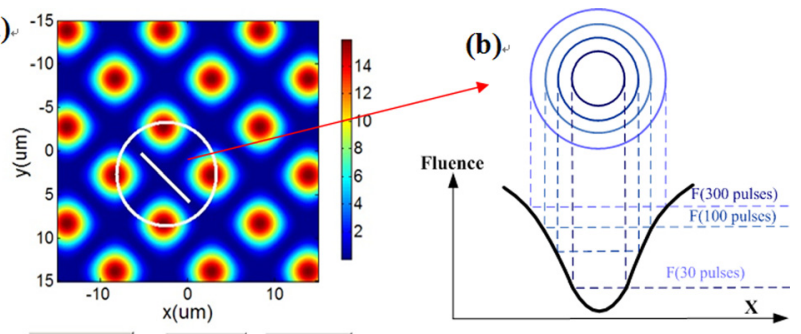

FIG. 11. (a) The computer simulation of intensity distribution of four-beam laser interference, the incident angle was $5.5^{\circ}$, the azimuthal angles were $0^{\circ}$, $90^{\circ}, 180^{\circ}$, and $270^{\circ}$, and the polarizers were $0^{\circ}, 90^{\circ}, 0^{\circ}$, and $90^{\circ}$ and (b) the schematic profile of a single negative peak dot intensity distribution after the effect of four-beam interference with the laser pulses of 30, 100, and 300 .

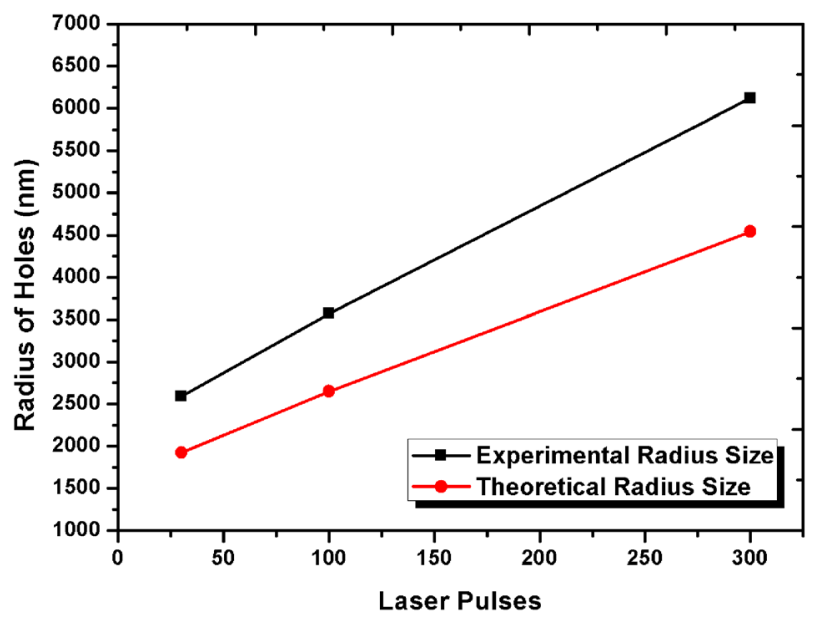

FIG. 12. Theoretical and experimental results for the comparison of their radius sizes with laser pulses.

In order to measure the reflectance, a Xenon-lamp, a spectrograph, and an integrating sphere with a detector were used. Fig. 13 shows that the reflectance of the pattern fabricated on the silicon wafer surface (blue curve) and the solarweighted reflectance $(S W R)$ is about $6.1 \%$ in the wavelength range from $380 \mathrm{~nm}$ to $780 \mathrm{~nm}$. The laser fluence was $637 \mathrm{~mJ} /$ $\mathrm{cm}^{2}$ with 300 laser pulses generated by four-beam laser

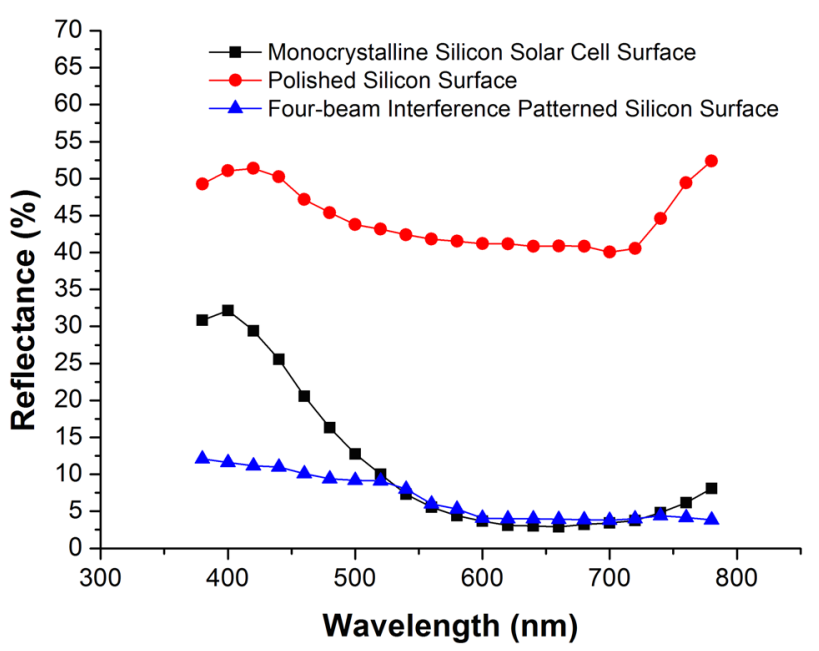

FIG. 13. The reflectance of the four-beam laser interference patterned silicon surface (blue curve), the monocrystalline silicon solar cell surface (black curve), and polished silicon surface (red curve). 
interference. For comparison, the reflectance of monocrystalline silicon solar cell surface (black curve in Fig. 13) and polished silicon surface (red curve in Fig. 13) were also measured and the SWRs were $8.9 \%$ and $43.8 \%$, respectively. Thus, one of the applications corresponding to the patterns fabricated by four-beam laser interference is the antireflection surface of solar cells, and it is important to understand the effects of laser fluence on silicon modification for the fabrication of well-defined surface structures in a four-beam laser interference process.

Compared with the focused single laser beam processing, four-beam laser interference lithography has the advantages of high peak fluences, high resolution, re-configurable patterns (feature sizes and shapes), and the creation of program controlled structures over large areas for the fabrication of periodic structures on various materials.

\section{CONCLUSIONS}

In this work, four-beam laser interference was used to pattern single crystal silicon wafers for the fabrication of surface structures, and the number of laser pulses was applied to the process in air. By controlling the parameters of laser irradiation, different shapes of silicon structures were obtained. The results were achieved with the single laser fluence of $354 \mathrm{~mJ} / \mathrm{cm}^{2}, 495 \mathrm{~mJ} / \mathrm{cm}^{2}$, and $637 \mathrm{~mJ} / \mathrm{cm}^{2}$, the pulse repetition rate of $10 \mathrm{~Hz}$, the laser exposure pulse of 30,100 , and 300 , the laser wavelength of $1064 \mathrm{~nm}$, and the pulse duration of 7-9 ns. The equations of heat flow and radiation effects of laser plasma of interfering patterns in a four-beam laser interference distribution were proposed to describe their impacts on silicon wafer surfaces. With the different laser fluence values of $354 \mathrm{~mJ} / \mathrm{cm}^{2}, 495 \mathrm{~mJ} / \mathrm{cm}^{2}$, and 637 $\mathrm{mJ} / \mathrm{cm}^{2}$, several physical processes such as the formation of flower-like holes, dots, and cones were observed. The results indicate that the laser fluence has to be properly selected for the fabrication of well-defined surface structures in a fourbeam laser interference lithography process.

\section{ACKNOWLEDGMENTS}

This work was supported by National Key Basic Research Program of China (973 Program No. 2012CB326406), EU FP7 (ECROBOT and BioRA), EU H2020 (FabSurfWAR), International Science and Technology Cooperation Program of China (No. 2012DFA11070), National Natural Science Foundation Program of China (Nos. 11504030, 60940035, and 61176002), Doctoral Program of Higher Education of China
(No. 20112216110002), Jilin Provincial Science and Technology Program (Nos. 201215136, 201115157, 20110704, and 20140414009GH), Guangdong Science and Technology Program (Nos. 2009B091300006 and2011B010700101), and Science and Technology Program of Changchun City (No. 11KP04).

${ }^{1}$ M. J. Sher, M. T. Winkler, and E. Mazur, MRS Bull. 36, 439-445 (2011).

${ }^{2}$ B. K. Nayak, M. C. Gupta, and K. W. Kolasinski, Appl. Surf. Sci 253, 6580-6583 (2007).

${ }^{3}$ M. Abbott and J. Cotter, Prog. Photovoltaics 14, 225-235 (2006).

${ }^{4}$ Y. Ma, H. Shi, J. Si, T. Chen, F. Yan, F. Chen, and X. Hou, Opt. Commun. 285, 140-142 (2012).

${ }^{5}$ J. Bonse, S. Baudach, J. Krüger, W. Kautek, and M. Lenzner, Appl. Phys. A 74, 19-25 (2002).

${ }^{6}$ J. Reif, O. Varlamova, M. Bounhalli, M. Muth, and T. Arguirov, Appl. Surf. Sci. 258, 9491-9495 (2012).

${ }^{7}$ X. Wang, Z. Shen, J. Lu, and X. Ni, J. Appl. Phys. 108, 033103-033107 (2010).

${ }^{8}$ B. Chichkov, C. Momma, S. Nolte, F. Von Alvensleben, and A. Tünnermann, Appl. Phys. A 63, 109-115 (1996).

${ }^{9}$ S. Nolte, C. Momma, H. Jacobs, A. Tünnermann, B. Chichkov, B. Wellegehausen, and H. Welling, J. Opt. Soc. Am. B 14, 2716-2722 (1997).

${ }^{10}$ A. Rodriguez, M. Echeverría, M. Ellman, N. Perez, Y. K. Verevkin, C. S. Peng, T. Berthou, Z. Wang, I. Ayerdi, and J. Savall, Microelectron. Eng. 86, 937-940 (2009).

${ }^{11}$ Z. Wang, J. Zhang, Z. Ji, M. Packianather, C. Peng, C. Tan, Y. Verevkin, S. Olaizola, T. Berthou, and S. Tisserand, in Proceedings of the $3 \mathrm{rd}$ International Conference on Manufacturing Engineering, 2008, pp. 929-936.

${ }^{12}$ Z. Zhang, Z. Wang, D. Wang, and Y. Ding, J. Laser Appl. 26, 012010 (2014).

${ }^{13}$ W. Zhao, J. Li, H. K. Kang, B. Zhou, and C. Wong, Nanosci. Nanotechnol. Lett. 3, 246-249 (2011).

${ }^{14}$ N. Pérez, T. Tavera, A. Rodríguez, M. Ellman, I. Ayerdi, and S. M. Olaizola, Appl. Surf. Sci. 258, 9370-9373 (2012).

${ }^{15}$ D. Wang, Z. Wang, Z. Zhang, Y. Yue, D. Li, R. Qiu, and C. Maple, J. Appl. Phys. 115, 233101 (2014).

${ }^{16}$ W. Li, Z. Wang, D. Wang, Z. Zhang, L. Zhao, D. Li, R. Qiu, and C. Maple, Opt. Eng. 53, 034109 (2014).

${ }^{17}$ V. V. Iyengar, B. K. Nayak, and M. C. Gupta, Sol. Energy Mater. Sol. Cells 94, 2251-2257 (2010).

${ }^{18}$ Z. Wang, J. Zhang, Z. Ji, M. Packianather, C. S. Peng, C. Tan, Y. K. Verevkin, S. M. Olaizola, T. Berthou, and S. Tisserand, in Proceedings of the 3rd International Conference on Manufacturing Engineering, 2008, pp. 929-936.

${ }^{19}$ D. P. Wang, Z. B. Wang, Z. Zhang, Y. Yue, D. Y. Li, and C. Maple, Appl. Phys. Lett. 8, 102 (2013).

${ }^{20}$ J. Han, W. Fan, Y. Li, L. Yang, G. Feng, X. Gao, Y. Liu, L. Bao, and Y. Huang, Pacific Rim Laser Damage Symposium: Optical Materials for High Power Lasers (International Society for Optics and Photonics, 2011), pp. 820616-820617.

${ }^{21}$ X. Chen, B. Bian, Z. Shen, J. Lu, and X. Ni, Microwave Opt. Technol. Lett. 38, 75-79 (2003).

${ }^{22}$ A. Hertwig, S. Martin, J. Kruger, and W. Kautek, Thin Solid Films 453, 527-530 (2004). 